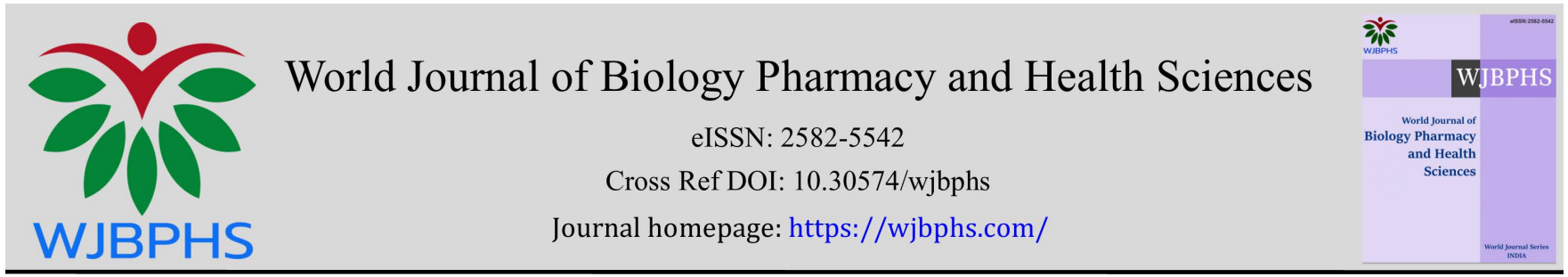

(RESEARCh ARTicle)

\title{
Antibiotic susceptibility pattern and plasmid profiles of nasal staphylococci from apparently healthy Nigerians
}

\section{DD Moro *}

Department of Microbiology, Lagos State University, P.M.B 0001, Ojo, Lagos, Nigeria.

World Journal of Biology Pharmacy and Health Sciences, 2021, 06(01), 019-025

Publication history: Received on 13 March 2021; revised on 15 April 2021; accepted on 17 April 2021

Article DOI: https://doi.org/10.30574/wjbphs.2021.6.1.0035

\begin{abstract}
A total of 480 nasal samples from apparently healthy Nigerian students were collected aseptically and analyzed bacteriologically. Staphylococci were recovered from 432 (90\%) of the subjects, constituting 288 (66.7\%) and 144 $(33.3 \%)$ of $S$. aureus and $S$. epidermidis respectively. The in-vitro antibiotic susceptibility testing using the disc diffusion technique showed high multiple resistance to the most commonly used antibiotics by Staphylococcus aureus such as penicillin (98.6\%), ampicillin (97.2\%), tetracycline (95.8\%) and streptomycin (84.7\%), but less resistance to erythromycin $(9.7 \%)$, rocephin $(8.3 \%)$, peflacin $(4.2 \%)$ respectively. The S. epidermis showed less resistance to all the antibiotics tested. Sixty percent of $S$. aureus harbored plasmids which molecular sizes ranged from 0.1 to 12.0 kilobases. The high prevalence of multiple antibiotic resistance appear to be plasmid mediated as plasmid profile analysis showed that about $90 \%$ of $S$. aureus harbored plasmids
\end{abstract}

Keywords: Staphylococci; S. aureus; S. epidermidis; Antibiotic susceptibility pattern; Plasmid Profile analysis

\section{Introduction}

Staphylococci are ubiquitous colonizers of human epithelial and mucous membrane [1]. The anterior nares are the primary ecological reservoir of staphylococcus aureus in humans and $S$. aureus nasal carriage is a major risk factor for a variety of infections [2]. Frequent carriage of $S$. aureus in the anterior nares have been consistently documented $[3,4,5]$. Staphylococcus aureus is the causative agent of many opportunistic infections in humans and animals [6]. S. aureus causes superficial deep-skin and soft tissue infections, endocarditis and bacteremia as well as toxin- mediated diseases including gastroenteritis, staphylococcal scalded skin syndrome and toxic shock syndrome [7]. Many pathogenic aerobic and anaerobic microorganisms from the environment often colonize different sites of the human body such as the nasopharynx, on the skin clothing or more rarely in the vagina $[8,9]$.

The nasal cavity is colonized by different pathways such as through hospital equipment, direct contact through lesions discharging pathogenic microorganisms [8,10]. According to Kluytmans [9], S. aureus has long been recognized as an important human pathogen and staphylococcal infections occur regularly in hospitalized patients with severe consequences despite antibiotic therapy. Almost everybody will have some type of staphylococcal infection during a lifetime which range in severity from food poisoning or minor skin infection to severe life-threatening infections [11].

Three human nasal S. aureus carriage patterns can be distinguished: persistent carriage, intermittent carriage and noncarriage. $S$. aureus density in the anterior nares is highest in persistent carriers, which will explain their increased risk of developing $S$. aureus infections [12].

\footnotetext{
* Corresponding author: Dauphin D. Moro, Ph.D

Department of Microbiology, Lagos State University, P.M.B 0001, Ojo, Lagos, Nigeria.
} 
S. aureus often possess the capacity to counter almost all the antimicrobial agents available to the clinicians for the treatment of severe staphylococcal infections [6,12]. Resistance to such antimicrobial compounds occur either through spontaneous mutation or are acquired through transmission from other related resistant bacteria [13,14]. Transfer of resistance through plasmids which contains genes for single of multiple antimicrobial agents have been reported in several bacterial pathogens including $S$. aureus [11,15]. However, resistance of staphylococci to a wide array of clinically useful antibiotics including streptomycin, rifampicin, fusidic acid and novobiocin has been attributed to chromosomal mutation [11]. Plasmid profiles have been of significance in epidemiological surveillance of disease outbreaks as well as in tracing sources of antibiotic resistance [12]. The possibility of transfer of bacteria harboring such plasmids from patients to apparently healthy individuals is not unlikely. The aim of the study is to isolate and identify Staphylococci from noses of apparently healthy undergraduates, assess the antibiotic susceptibility pattern, screen $S$. aureus for plasmids and determine any relationship between antimicrobial resistance of staphylococci and carriage of plasmids.

\section{Material and methods}

\subsection{Sample Population}

Four hundred and eight apparently healthy students of the Faculty of Science, Lagos State University, Ojo Campus were randomly screened for nasal carriage of staphylococci after obtained consent from students of each of the three Departments: Biochemistry, Botany and Microbiology.

\subsection{Biological Analysis of Samples}

Four hundred and eighty nasal swabs from the students were examined bacteriologically between January 2017 and December, 2018. Each swab stick (Even pore) was moistened with a sterile distilled water prior to introduction into the nose and was streaked - inoculated directly into sterile blood agar and Staphylococcus 110 agars (Oxoi, Basingoke, U.K). The plates which were in duplicates were then incubated aerobically and at $5 \% \mathrm{CO}_{2}$ for 24 hours respectively. Staphylococci were identified on the basis of colonial, morphological and biochemical tests such as Gram staining, presence of coagulase positive cocci in clumps, the detection of coagulase production with fresh rabbit plasma and fermentation of mannitol [16].

\subsection{Antibiotic Susceptibility Testing}

The $S$. aureus were tested for the susceptibility to ten different antimicrobial agents using the disc diffusion technique [17]. Bacterial strains inoculated into Mueller-Hinton broth (Oxoid, Basingstoke, U.K) and incubated at $37^{\circ} \mathrm{C}$ for $18-$ $24 \mathrm{hrs}$. the inoculum was standardized by adjusting the turbidity of an actively growing broth culture to match the barium sulphate (0.5 McFarland) standard. Mueller-Hinton agar plates (Oxoid, Basingoke, U.K) were then inoculated with bacterial suspensions and spread as evenly as possible. Standard sensitivity discs with the following concentration were introduced with a sterile forceps: Penicillin $(10 \mu \mathrm{g})$, Amoxicillin $(10 \mu \mathrm{g})$, chloramphenicol (50 $\mu \mathrm{g})$, erythromycin $(15 \mu \mathrm{g})$, gentamicin $(10 \mu \mathrm{g})$, tetracycline $(30 \mu \mathrm{g})$, streptomycin $(10 \mu \mathrm{g})$, cloxacillin $(10 \mu \mathrm{g})$, Rocephin $(10 \mu \mathrm{g})$ and peflacin $(10 \mu \mathrm{g})$ and incubated at $37^{\circ} \mathrm{C}$ for $18-24 \mathrm{hrs}$. After incubation, the diameter of the zone of inhibition was measured to the nearest millimeter and interpreted $[17,18]$. Staphylococcus aureus strain ATCC 25923 was used as control.

\section{Plasmid profile analysis}

Plasmid DNA was isolated by the technique of Birnboim and Doly [19] modified by the use of lysostaphin for lysing the cells. Each $S$. aureus isolate was inoculated into $3 \mathrm{ml}$ tryptic soy broth and incubated overnight on a roller drum at $37^{\circ} \mathrm{C}$. About $1.5 \mathrm{ml}$ of each overnight broth culture was transferred into Eppendorf tubes and centrifuged for $1 \mathrm{~min}$ at 5,000 $\mathrm{rpm}$ at room temperature. The supernatant was discarded and $2 \mu \mathrm{l}$ of lysostaphin solution $(1.0 \mu \mathrm{g} / \mathrm{ml}$ in distilled water $)$ added to the pellet. Tubes were capped, vortexed and placed in ice for $30 \mathrm{~min}$. $200 \mu \mathrm{l}$ of alkaline detergent $(0.2 \mathrm{NaOH} ; 1 \%$ SDS) was added and the tubes were inverted several times and kept in the water bath for $5 \mathrm{~min}$. $150 \mu \mathrm{l}$ of $3 \mathrm{M}$ sodium acetate ( $\mathrm{pH} 4.8)$ was added and the tubes inverted several times to mix and kept iced for at least 10 mins. The tubes were centrifuged at room temperature at 15,000 rpm for $5 \mathrm{~min}$ and the supernatant transferred into new Eppendorf tubes. $1 \mathrm{ml}$ of $95 \%$ ice cold ethanol was added to the test tubes which were kept at $-20^{\circ} \mathrm{C}$ for $5 \mathrm{~min}$. After $5 \mathrm{~min}$, they were centrifuged at $1500 \mathrm{rpm}$ for 3mins; supernatant discarded and the sediment resuspended in $50 \mu \mathrm{l}$ of Tris/EDTA (10Mm Tris HCL and 1Mm EDTA pH 8.0)

\subsection{Agarose gel electrophoresis of plasmid DNA.}

Plasmid DNA electrophoresis was carried out in a 0.7\% agarose gel (sigma) with Tris-borate-EDTA buffer pH 8.3 [19] at $45 \mathrm{Ma}$ for $2 \mathrm{~h}$ at room temperature using an LKB vertical electrophoresis apparatus (LKB, Bromma). The gels were 
stained with ethidium bromide (sigma) for $20 \mathrm{~min}$ and then photographed with Polaroid 665 film under short wave UV light through a red filter.

\section{Results}

A total of 432 staphylococci were identified comprising 288 (66.7\%) S. aureus and 144 (33.3\%), S. epidermis. A high frequently of resistance by $S$. aureus to penicillin (98.6\%), ampicillin (97.22\%), tetracycline (95.8\%), streptomycin $(84.7 \%)$ and chloramphenicol (55.6\%) was observed. The staphylococci however showed susceptibility to cloxacillin, gentamicin, erythromycin, Rocephin and peflacin (Table 1).

The $S$. aureus isolates were grouped into eleven plasmid profile groups on the basis of the number and molecular weights of the plasmid they harbor. The number of plasmids per isolate ranged between 3 to 6 but most isolates had four plasmids each. The molecular sizes of the plasmids $(0.7,1.4,1.6 \mathrm{~kb})$ most commonly encountered (Table 2$)$. The $S$. aureus which showed very high multiple resistance to many antibiotics had more plasmid with high molecular sizes. Multiple resistance decreased with decreasing plasmid size.

Table 1 Antibiotic Resistance pattern of staphylococci from Nigerian students

\begin{tabular}{|l|l|l|}
\hline Antimicrobial agent & S. aureus $\mathbf{N = 2 8 8 ( \% )}$ & S. epidermis $\mathbf{N = 1 4 4 ( \% )}$ \\
\hline Ampicillin & $280(97.2)$ & 66.7 \\
\hline Penicillin & $282(95.8)$ & $104(72.2)$ \\
\hline Tetracycline & $276(95.8)$ & $96(66.7)$ \\
\hline Streptomycin & $244(84.7)$ & $76(52.8)$ \\
\hline Chloramphenicol & $160(55.6)$ & $56(38.9)$ \\
\hline Cloxacillin & $44(15.3)$ & $16(11.1)$ \\
\hline Gentamycin & $36(12.5)$ & $16(11.1)$ \\
\hline Erythromycin & $28(9.7)$ & $12(8.3)$ \\
\hline Rocephin & $24(8.3)$ & $16(11.1)$ \\
\hline Peflacin & $12(4.2)$ & $8(5.6)$ \\
\hline
\end{tabular}

Table 2 Antibiotic Resistance Pattern and Plasmid Profile of $S$. aureus

\begin{tabular}{|l|l|l|}
\hline Antibiotic resistance pattern & No (no=60) & Plasmid size \\
\hline A C Cl E G Pf Pn R S T & 13 & $1.0,1.5,6.0,11.0,12.5$ \\
\hline A C Cl E G Pn R S T & 8 & $1.0,1.4,3.5,10.0,12.0$ \\
\hline A C Cl E G Pn S T & 7 & $1.0,1.5,3.5,6.0$ \\
\hline A C Cl G Pn S T & 6 & $1.0,1.5,1.8,2.8,5.5$ \\
\hline A C Cl Pn S T & 6 & $0.7,1.4,1.6,4.0$ \\
\hline A C Pn S T & 4 & $0.7,1.6,4.0$ \\
\hline A Pn S T & 2 & $1.0,1.6,2.0,2.5$ \\
\hline A Pn T & 3 & $1.0,1.8,2.5,2.8$ \\
\hline A Pn & 5 & $0.7,1.2,1.6,1.8$ \\
\hline 0 & 6 & None \\
\hline \multicolumn{2}{|c|}{ A - Ampicillin, C- chloramphenicol, Cl-cloxacillin, E- erythromycin, G-Gentamycin, } \\
Pf - Peflacin, Pn - Penicillin R- Rocephin, S - streptomycin, T- Tetracycline.
\end{tabular}




\section{Discussion}

Several microorganisms have been reported to contaminate some sites on the skin or mucous membrane and other part by interpersonal transfer by air or by direct contact [8]. However, the nasal cavity can be contaminated through handling of specimens, direct contact with a carrier or contact with unsterilized hospital equipment or contact with infected animals [20].

This study revealed a high nasal carriage of $S$. aureus constituting $66.7 \%$ of the 432 bacterial isolates from apparently healthy undergraduates studied. The high prevalence of $S$. aureus observed in this study underscores its significance as this organism has been implicated in several human diseases such as skin abscess, endocarditis, osteomyelitis, meningitis etc. [21]. This finding agrees with an earlier report by Kluytmans [9], that a large proportion of apparently healthy population harbor $S$. aureus intermittently. Routine assessment of the carrier rate of $S$. aureus on patient particularly those with skin puncture such as hemodialysis, continuous ambulatory dialysis (CAPD), intravenous drug users, and even in human immunodeficiency virus patients are therefore important [9]. This finding also conforms with that of Lamikanra [22], who reported a higher prevalence of $S$. aureus from apparently healthy Nigerian students. However, the findings in this study are significantly higher than other studies cited by Rodoguez et al. [24] wherein rates ranged from 7.6 to $53.8 \%$. the substantial proportion of staphylococci isolates in a non-nosocomial setting as identified in this study are of great concern.

The recovery of S. epidermidis from 33.3\% of the undergraduates is of serious health concern as S. epidermidis has been reported to possess virulent factors often similar to that of $S$. aureus, thus may be more intrinsically virulent than previously thought [23]. A similar finding with higher rate of S. aureus as against S. epidermis was reported [22].

There were no significant differences in age and sex distribution of students sampled, majority of female and male who harbor $S$. aureus fell within the 20 to 25 age range with frequencies of $52.8 \%$ and $47.2 \%$ in females and males respectively.

Wang [26], reported no significant differences in the age and certain behaviors as well as the environment where the person work or live. Nawa's and Fakhoury [15] however, reported a high carrier rate of S. aureus among hospital staff.

The high frequency of in-vitro resistance demonstrated by $S$. aureus against the commonly used antibiotics like penicillin, chloramphenicol, tetracycline and streptomycin is quite astonishing. A high carrier rate of $S$. aureus with a much as $90 \%$ resistance to penicillin has been reported $[8,25]$.

Since different strains of $S$. aureus differ in sensitivity to different antibiotics, the choice of antibiotic in the treatment of patients should be based on sensitivity tests made on isolates from patients [22]. Resistant bacteria may transfer resistance genes to other bacteria hence become important in the spread of antibiotic resistance [12]. Indiscriminate use of antimicrobial agents and antibiotics sale such as sale of antibiotics without prescription, sale of under-dose and substituting brands enhances development of drug resistance [25]. The high occurrence of multiple antibiotics resistance observed in the staphylococci studied may be due to abuse of such drugs in the community and previous exposure of the subject to the antibiotics as earlier reported by Merhase [29]. The dominant role played by $S$. aureus in post-operative wound infections, which source is usually endogenous is also likely among our subjects. These findings agree with earlier reports by several authors such as Kluytmans et al., [9], from 20\% upwards, Perez-Fortan et al., [30] with $30-7-\%, 40 \%$ by Joklik et al., [31], and $40-50 \%$ carrier rate of $S$. aureus by Jawetz [22]. This underscores the need to screen individuals for nasal carriage of $S$. aureus routinely during medical examination. A compulsory preoperative procedure of nasal carriage evaluation of patients is hereby strongly advocated.

Nasal culture has been acknowledged as being a suitable source for the detection of $S$. aureus as the anterior important site of colonization and source of dissemination of $S$. aureus thus associated with a higher rate of staphylococcal infection [14].

The frequency of sampling has been reported to affect the rate of colonization especially among medical staff caring for possible multiple antibiotic resistant $S$. aureus (MARSA), patients which may be transient, short-term or persistent as a single random sampling of subjects may not give the precise rate of colonization with S. aureus [30]. Several reports however suggested that colonization may occur at different sites of the body, so culture of multiple sites has been recommended in order to ensure detection of carriage of MARSA particularly methicillin resistant S. aureus (MARSA) [14]. In addition, carriage of MARSA has been further compounded by the economic downturn leading to low patients' turnout in hospitals who often patronize traditional medical practitioners alternatively [31]. 
The lowest prevalence of $S$. aureus observed among the microbiology students can be attributed to the aseptic conditions as well as consciousness of contaminants while working in the laboratory as against other students.

Plasmid profile have been reported to be useful in tracing the epidemiology of antibiotic resistance [12]. It was observed that $85 \%$ of the $60 \mathrm{~S}$. aureus studied which were multiple antibiotic resistant harbored plasmids. In addition, the $S$. aureus isolates which harbored large molecular size plasmids exhibited high multiple antibiotic resistance thus suggests that plasmid is likely to play a major role in the antibiotic resistance of $S$. aureus. However, it was observed in this study that resistance to various antibiotics was not associated with the presence of specific plasmid or genes. Resistance to antibiotics by $S$. aureus may be likely located either on the chromosomes, plasmids, transposons or integron [11]. $S$. aureus which have plasmids ranging from 5.0 to $12.5 \mathrm{~Kb}$ were resistant to between 5 to 10 different antibiotics. We were however, unable to isolate and relate specific plasmid to specific antibiotic.

\section{Conclusion}

The high nasal carriage of staphylococci was observed among apparently healthy Nigerians. More of the staphylococci were $S$. aureus and less of S. epidermis. S. aureus were multiply resistant to commonly used antibiotics and susceptible to the less commonly used and more expensive antibiotics. Multiple resistance in $S$. aureus is likely to be plasmid mediated as $S$. aureus resistant to 5 to 10 different antibiotics had plasmids which ranged from 5.0 to $12.5 \mathrm{~kb}$. Further studies on nasal carriage of methicillin-resistant staphylococci (MRSA) is strongly recommended.

\section{Compliance with ethical standards}

\section{Acknowledgments}

The author wholeheartedly appreciates several laboratory staff in the Department of Microbiology, Ojo Campus who prepared the media, analyzed the sample and helped reading the plates under my supervision. The contributions of the staff of Genetics Division, Nigerian Institute of Medical Research (NIMR), Yaba, Lagos., in the isolation of the plasmid DNA while separating same from chromosomal DNA is sincerely appreciated. Mr. Chinedum Emmanuel Usuah is highly appreciated for typing and formatting this manuscript.

\section{Disclosure of conflict of interest}

Not applicable.

\section{Disclosure of informed of consent}

The subjects were informed of the purpose and significance of the study, so only volunteers who gave informed consent were recruited for the study while those who declined were excluded.

\section{References}

[1] Hamdan-Partida A, Sainz-Espunes T, Bustos-Martinez J. Isolation of community acquired methicillin-resistant staphylococcus aureus in health carriers in a Mexican community. Int. J. Infect. Disease. 2014; 18:22-26.

[2] Wertheim HF, Melles DC, Vos MC. The role of nasal carriage in S. aureus infections. Lancet Infect. Dis 2005; 5:75162.

[3] Veira M.A, Minamisava R, Pesoa-Junior V, Lamaro-Cardso J, Ternes Y, Andre M.C. Methicillin-resistant staph aureus nasal carriage in neonates and children attending a paediatric outpatient clinic in Brazil. Braz. J. Infect. Dis. 2014;18(1):42-47.

[4] Maratas L, Cuadra J, Nasal carriage of S. aureus and Methicillin-resistant S. aureus among healthy children of Higan city, Philiphines. Int. J. Bios. 2017;5:140-145.

[5] Erica LJ, Van Den Akker, Nouwen JL, Melles DC, Rossum EFC, Koper JW, Alitheriden AG, Hofman A, Verburgh HA, Pols HA, Lamberts SWJ, Belkum AV. Infect. Dis 2006;194:814-818.

[6] Kools WE, Bannerman TL. Staphylococcus and Micrococcus In: Murray, P.R., Baron, E. J, Pfaller, M.A., Tenover, F.O, Yolken, R.H (Ed). Manual of Clinical Microbiology, 6 Ed. American Society for Microbiology, Washington D.S. $1995 ; 282-298$. 
[7] Fidalggo ST, Vasgues F, Mendaza MC, Perez J, Mendez FJ. Bacteraemia due to Staphylococcus epidermidis. microbiological, clinical and prognostic Features. Rev. Infect.Dis. 1990; 12: 520 - 528.

[8] Mackie JP, Duguid BP, Marima RH. A Medical Microbiology. Churchill Livingstone, New York. $1989 ; 254$ - 260.

[9] Kluytmans J. Reduction of Surgical Site Infection in Major Surgery by Elimination of Nasal Carriage of Staphylococcus aureus. J. Hosp. Infect. 1998; 10: 25 - 29.

[10] Heczko PB, Bulanda M,Hoeffler U. Nasal carriage of Staphylococcus aureus and its influencw on Hospital Infection caused by Methicillin-resistant strains. Zbl. Bakt. 1990;274:333-341.

[11] Hayanga A, Okello A, Hussein R, Nyong' O. An Experience With Methicillin Resistant Staphylococcus aureus At Nairobi Hospital. East Afr. Med. J. 1997; 74: 203 -204.

[12] Nouwen IL, Ott A, Kluytmans-Nndenbergh MF. Predicting the S. aureus nasal carrier statte derivation and validation a 'culture rule'. Clin. Infect. Dis. 2004;39:806-11.

[13] Ombui JN, Kimotho AM, Nduhiu JG. Antimicrobial Resistance Patterns and Plasmid Profiles of Staphylococcus aureus Isolated from Milk and Meat. East. Afr. Med. J. 2000; 77: 461 - 467.

[14] Clewel BD. Plasmid Drug Resistance and Gene Transfer in The Genus Streptococcus. Microbiol. Rev. 1981; 45: 409.

[15] Nawa's T, Fakhoury J. Nasal Carriage of Methicillin - Resistant Staphylococcus aureus by Hospital Staff in North Jordan. J. Hosp. Infect. 1991; 17: 223 - 229.

[16] Locksley RM, Cohen ML, Quinn TC, Tompkins LS, Coyle BM, Kinhara JM, Counts GN. Multiply Antibiotic Resistant Staphylococcus Aureus: Introduction, Transmission and Evolution of Nosocomial Infection. Ann. Intern. Med. 1982; 97: $317-324$.

[17] Barrow GI, Feltham RKA. Cowan and Steel'S Manual for The Identification of Medical Bacteria. Pp 52 - 58. Cambridge University Press. 1995.

[18] National Committee for Clinical Laboratory Standards: Performance Standard for Antimicrobial Disc Susceptibility Test. Approved Standard M2 - A6, 8th International Supplement. Wayne. 1998.

[19] Birnboim HC, Doly J. A Rapid Alkaline Extraction Procedure for Screening Recombinant Plasmid Dna. Nucleic Acids Res. 7: 1513 - 1523.

[20] Meyers JA, Sanchez D, Elwell LP, Falkow S. Simple Agarose Gel Electrophoresis Method for The identification and characterization of plasmid Deoxyribonucleic Acid J. Bacteriolo. 1976; 127: 1529 - 1537.

[21] Casewell MW. The Nose; An Underestimated Source of Staphylococcus aureus Causing Wound Infection. J. Hosp. Infect. 1998; 40: 3 - 11.

[22] Jawetz E, Melnick JL, Adelberg EA. Medical Microbiology. Prentice - Hall Int. Inc. New Jersey. $1998 ; 202$ - 212.

[23] Laamikanra A, Ogunsanya OTA. Long Study of Nasal Carriage of Staphylococcus aureus In a population Of Healthy Nigerian Students, Nig. J. Microbiol. 1982; 6: 5 - 7.

[24] Rodriguez EA, Correa MM, Ospina S, Atehortua SL, Jimenez JN. Differences in epidemiological and molecular characteristics of nasal colonization with S. aureus(MSSA-MRSA) in children from a University Hospital and Day care centers. PLoS One. 2014;9(7):12-24

[25] Gemmel CG, Roberts CE. Virulence Characteristics of Staphylococcus epidermis. J.Med. Microbiol. 1986; $22: 520$ -528 .

[26] Wang JT, Chang SC, Kot WJ, Chang YY, Chens ML, Pans HJ, Luh KT. A hospital Acquired Outbreak of Methicillin Resistant Staphylococcus aureus Initiated by A Surgeon Carrier. J. Hosp, Infect. 2001; 47: 104 - 109.

[27] Ogunsola FT. Penicillin - Resistant Pneumococcus. J. Hosp. Med. 1997; 7: 120 - 128.

[28] Indalo A. An Antibiotic Sale Behaiviour In Nairobi: A Contributing Factor To Antimicrobial Drug Resistance. East Afr. Med. J. 1997; 74: 171 - 173.

[29] Merhase A, Debbia EA, Arvigo A, Pesce A, Schito GC. Susceptibility of Susceptibility of Streptococcus Pneumoniae Strains Isolated in Italy To Penicillin and Ten Other Antibiotics, J. Antimicro. Chemother. 1995; $36: 833$ - 837. 
[30] Perez-Fontan M, Rosales M, Rodriguez- Carmona A. Treatment of Staphylococcus aureus Nasal Carrier in Continuous Ambulatory Peritoneal Dialysis with Mupirocin: Long-Term Result. Am. J. Kidney Dis. 1992; 22: 708 $-712$.

[31] Joklik WK, Willet HP, Amos B. Zinsser Microbiology, 20th Ed. Connecticut: Appleton And Lange. 1992.

[32] Cookson B, Webster M, Peter B, Philip I, Rahman M, Noble W. Staff carriage of endemic methicillin resistance Staphylococcus aureus. J. Clin. Microbial. 1989; 27: 1471 - 1475.

[33] Daniel SO. An epidemiological study of nosocomial infections at the Lagos State University Teaching Hospital. Community Health. 1997; 8: 139 - 144. 